\title{
Use of Social Media to Promote Cancer Screening and Early Diagnosis: Scoping Review
}

Ruth Plackett ${ }^{1}, \mathrm{PhD}$; Aradhna Kaushal ${ }^{2}, \mathrm{PhD}$; Angelos P Kassianos ${ }^{1}, \mathrm{PhD}$; Aaron $\mathrm{Cross}^{2}$, MSc; Douglas Lewins ${ }^{3}$; Jessica Sheringham ${ }^{1}, \mathrm{PhD}$; Jo Waller ${ }^{4}, \mathrm{PhD}$; Christian von Wagner ${ }^{2}, \mathrm{PhD}$

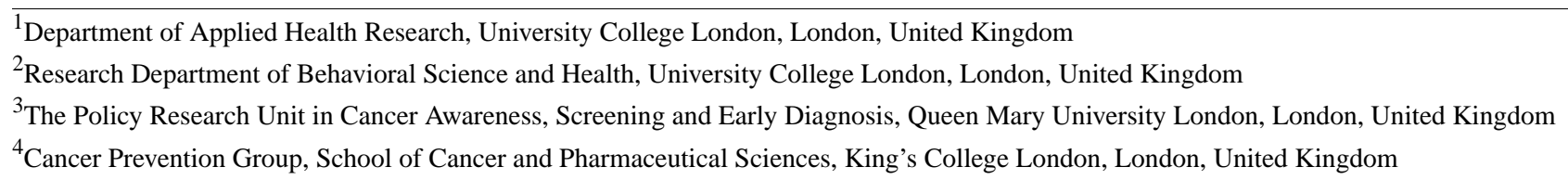

Corresponding Author:

Ruth Plackett, $\mathrm{PhD}$

Department of Applied Health Research, University College London

1-19 Torrington Place,

Bloomsbury

London, WC1E 7HB

United Kingdom

Phone: 442031083242

Email: ruth.plackett.15@ucl.ac.uk

\section{Abstract}

Background: Social media is commonly used in public health interventions to promote cancer screening and early diagnosis, as it can rapidly deliver targeted public health messages to large numbers of people. However, there is currently little understanding of the breadth of social media interventions and evaluations, whether they are effective, and how they might improve outcomes.

Objective: This scoping review aimed to map the evidence for social media interventions to improve cancer screening and early diagnosis, including their impact on behavior change and how they facilitate behavior change.

Methods: Five databases and the grey literature were searched to identify qualitative and quantitative evaluations of social media interventions targeting cancer screening and early diagnosis. Two reviewers independently reviewed each abstract. Data extraction was carried out by one author and verified by a second author. Data on engagement was extracted using an adapted version of the key performance indicators and metrics related to social media use in health promotion. Insights, exposure, reach, and differing levels of engagement, including behavior change, were measured. The behavior change technique taxonomy was used to identify how interventions facilitated behavior change.

Results: Of the 23 publications and reports included, the majority $(16 / 23,70 \%)$ evaluated national cancer awareness campaigns (eg, breast cancer awareness month). Most interventions delivered information via Twitter (13/23, 57\%), targeted breast cancer $(12 / 23,52 \%)$, and measured exposure, reach, and low- to medium-level user engagement, such as number of likes $(9 / 23,39 \%)$. There were fewer articles about colorectal and lung cancer than about breast and prostate cancer campaigns. One study found that interventions had less reach and engagement from ethnic minority groups. A small number of articles $(5 / 23,22 \%)$ suggested that some types of social media interventions might improve high-level engagement, such as intended and actual uptake of screening. Behavior change techniques, such as providing social support and emphasizing the consequences of cancer, were used to engage users. Many national campaigns delivered fundraising messages rather than actionable health messages.

Conclusions: The limited evidence suggests that social media interventions may improve cancer screening and early diagnosis. Use of evaluation frameworks for social media interventions could help researchers plan more robust evaluations that measure behavior change. We need a greater understanding of who engages with these interventions to know whether social media can be used to reduce some health inequalities in cancer screening and early diagnosis.

International Registered Report Identifier (IRRID)： RR2-10.1136/bmjopen-2019-033592 


\section{KEYWORDS}

social media; review; cancer; campaign; health promotion; public health; early detection of cancer; cancer screening; health care disparities

\section{Introduction}

Social media is becoming increasingly popular, estimated to be used by well over half of the world's population [1,2]. Given social media's potential for widespread public engagement, it is commonly incorporated into public health interventions $[3,4]$. The term "intervention" in this article refers to a spectrum of activities ranging from national awareness-raising public health campaigns, such as breast cancer awareness month (BCAM), to more targeted activities that use social media functions like discussion groups to deliver information to specific audiences. Social media interventions can influence behavior by targeting cognitive or emotional responses, generating discussions, and changing social norms [5]. Additionally, social media allows health messages to be disseminated rapidly, at low cost, to large numbers of people across large geographical areas [6-12].

Several national public health campaigns have used social media to try to improve the early diagnosis of cancer through raising awareness of cancer symptoms, encouraging help-seeking, and attempting to influence social norms around help-seeking $[13,14]$. However, there are concerns that the effects of these campaigns are short-lived, often only involve one-way communication, and do not benefit those in most need $[4,5,15,16]$. Social media can be used to target messages toward specific geographical regions or demographic groups, such as those who are known to have poor knowledge of cancer symptoms or encounter more barriers to accessing cancer screening $[17,18]$. Therefore, social media interventions may be more able to address health inequalities than traditional interventions $[9,19,20]$. However, little is known about the unintended effects of social media interventions and the possibility of spreading misinformation [9,21,22]. Additionally, social media cannot reach those with poor access to digital technology, who may also have the greatest need for public health information [23].

Despite increased use of social media interventions, there is little evidence about whether they improve cancer screening and early diagnosis [24]. There are no review publications that describe the variety of ways that social media specifically is used to promote cancer screening and early diagnosis, how these interventions might facilitate behavior change, and how this has been evaluated. Previous systematic reviews looking at a range of different media-based interventions for cancer screening included very few articles evaluating social networking sites like Facebook and have focused on specific research questions about impact and effectiveness [25,26]. However, use of social media interventions for public health is evolving rapidly and there is a need for a broader mapping of diverse studies to inform future development and evaluations. Therefore, a scoping review methodology was used to map the literature on the ways in which social media has been used to promote cancer screening and early diagnosis and how it was evaluated [27]. Specifically, we aimed to address the following research questions: (1) What are the characteristics of social media interventions that aim to promote cancer screening and early diagnosis?, (2) What are the mechanisms of change by which these interventions promote behavior change?, (3) What methodological approaches have been used to evaluate interventions?, (4) What are the outcomes used to measure the impact of interventions?, and (5) What are the key findings?

\section{Methods}

\section{Overview}

Social media interventions designed to promote cancer awareness and screening were identified using a scoping review guided by the methodology of Arksey and O'Malley [28] and expanded upon by Levac et al [29] and Peters et al [30]. We followed the process outlined in the published protocol and followed the Preferred Reporting Items for Systematic reviews and Meta-Analyses extension for Scoping Reviews (PRISMA-ScR) [31,32].

\section{Search Strategy}

An experienced research librarian helped to develop the search strategy, which included a combination of subject headings and keyword searches. We identified articles by searching five databases: MEDLINE, PsycINFO, Scopus, Web of Science, and CINAHL (Cumulative Index to Nursing and Allied Health Literature). Multimedia Appendix 1 shows the full search strategy used for MEDLINE, which was adapted for the other databases. Additional articles were identified by conducting internet searches for relevant published material and by hand-searching reference lists of included articles. We searched the grey literature for relevant reports not published in peer-reviewed journals. We also contacted the authors of conference abstracts that met inclusion criteria to see if we could include any unpublished results. Organizations and charities related to cancer screening were contacted via email for any relevant published research reports.

\section{Inclusion and Exclusion Criteria}

We considered peer-reviewed articles and non-peer-reviewed reports from the grey literature, as this was a scoping review that aimed to be inclusive and to explore the breadth of relevant research. The findings will help to inform future systematic reviews of the literature that will also assess the quality of the research. Additionally, non-peer-reviewed articles were included because many evaluations of social media interventions are not published in peer-reviewed journals. Social media is also a rapidly evolving area and current insights might not be captured in peer-reviewed literature, where there is typically a lag time between evaluation and publication. We excluded articles and reports not published in English, as we could not feasibly translate the results into English in a valid and reliable way. Articles and reports that discussed social media platforms, including Facebook, Twitter, Instagram, YouTube, Pinterest, and Snapchat, were eligible for inclusion. Articles relating to social media platforms popular in non-English-speaking 
countries were not excluded, but as a consequence of limiting our search to articles written in English, the focus of this review is on platforms commonly used in English-speaking countries. We included articles published from 2004-as this was the advent of widespread social media use of these platforms-to June 2019 [33]. The reported findings from mass media campaigns were included if social media was the primary focus of the article. We included articles that discussed both qualitative and quantitative methods. We only included articles about interventions that directly targeted cancer screening and early diagnosis, where the primary message of the intervention focused on raising awareness of cancer symptoms, cancer screening, or promotion of help-seeking for potential cancer symptoms. Articles that reported findings solely on the human papillomavirus vaccine were excluded, as wider issues regarding attitudes toward vaccination and misinformation were considered out of the scope of this review. We excluded articles if the participants were cancer patients, survivors, or health professionals. Articles that evaluated static internet pages, such as blog posts, were also excluded from the review.

\section{Article Screening and Data Extraction}

All identified articles underwent two stages of screening: title/abstract screening and full-text screening. Three researchers (APK, AC, and SR) divided the articles and screened them against the inclusion criteria. Each article was independently reviewed by another researcher (AK). If the eligibility of any title/abstract was unclear, it was included in the full-text screening and any discrepancies were reviewed by an additional author and resolved in a consensus meeting. Interrater reliability for title/abstract screening was good (Cohen $\kappa=0.69)$ [34]. Two researchers (RP and $\mathrm{AK}$ ) piloted the data extraction approach and three researchers (RP, APK, and AC) completed the data extraction. We extracted data using a Microsoft Access database to collect key information on article characteristics, details of the interventions, methodological approaches, outcome measures, and key findings. A second researcher (RP, APK, or AC) verified the data extractions, and discrepancies were resolved in regular meetings with the entire team.

Table 1 describes the outcomes extracted from the included articles. These were categorized using an adapted version of Neiger et al's [35] key performance indicators and metrics related to social media use in health promotion, which included insights, exposure, and reach, as well as low-, medium- and high-level engagement. Outcome measures capturing cancer knowledge and intention to attend cancer screening were not accounted for using the original framework but were categorized as high-level engagement, as greater knowledge and intentions are precursors to behavior change $[36,37]$. We also extracted information on the nature of information that was delivered and shared in interventions by users and developers, such as how many posts were related to health issues and fundraising. Additional data extraction was undertaken by one researcher (RP) to understand the mechanisms by which interventions might promote behavior change by using the behavior change technique (BCT) taxonomy developed by Michie et al [38]. BCTs were identified from the articles but also from campaign websites where possible. Following the scoping review methodology [28-30], we consulted with six people with experience developing and evaluating social media interventions for cancer screening, as well as a cancer patient, to validate our findings [39]. We asked them to comment on our preliminary findings, and their input helped to shape the narrative synthesis of the data.

Table 1. Descriptions and examples of outcomes captured during data charting. ${ }^{\text {a }}$

\begin{tabular}{|c|c|c|}
\hline Data charting outcome & Description & Examples \\
\hline Nature of information delivered/shared & $\begin{array}{l}\text { Type of messages delivered by intervention or } \\
\text { shared by users }\end{array}$ & $\begin{array}{l}\text { Number of messages referring to } \\
\text { cancer }\end{array}$ \\
\hline Insights & User feedback & Users' opinions of information \\
\hline Exposure & Views of social media content & Number of views of posts/tweets \\
\hline Reach & $\begin{array}{l}\text { Interaction with social media content and users' } \\
\text { characteristics }\end{array}$ & $\begin{array}{l}\text { Number of page likes and demo- } \\
\text { graphics of users }\end{array}$ \\
\hline Low-level engagement & Agreement with the social media content & Number of likes of posts \\
\hline Medium-level engagement & $\begin{array}{l}\text { Users creating or sharing their own social me- } \\
\text { dia messages or sharing intervention messages } \\
\text { on their own profiles }\end{array}$ & Number of posts/retweets \\
\hline High-level engagement (behavior change) & $\begin{array}{l}\text { Users' understanding of the messaging, inten- } \\
\text { tion to change their behavior, or actions taken } \\
\text { offline related to the desired behavior change }\end{array}$ & $\begin{array}{l}\text { Change in cancer screening atten- } \\
\text { dance }\end{array}$ \\
\hline
\end{tabular}

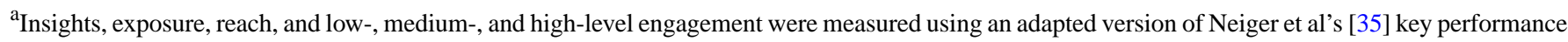
indicators and metrics related to social media use in health promotion. 


\section{Results}

\section{Article Selection}

Based on the initial search, 1029 articles were identified after duplicates were removed. We screened a total of 183 full-text articles and included 23 of those articles in this review. Figure 1 outlines the selection process.

Figure 1. Preferred Reporting Items for Systematic reviews and Meta-Analyses extension for Scoping Reviews (PRISMA-ScR) flow diagram of the literature search and article selection process.

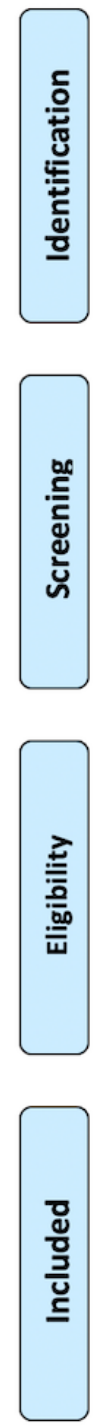

\section{Characteristics of Articles}

Multimedia Appendix 2 outlines the characteristics of each included article [40-62]. Only 2 of 23 (9\%) articles were from the grey literature and were charity campaign reports; the rest of the articles were published in academic journals. First author institutions represented 10 countries, most commonly the United States $(11 / 23,48 \%)$, Canada $(3 / 23,13 \%)$, and the United Kingdom $(2 / 23,9 \%)$.

\section{Characteristics of Social Media Interventions}

\section{Types of Interventions}

Multimedia Appendix 2 describes the characteristics of each intervention. We found four different types of social media interventions present in the literature: (1) national cancer awareness month campaigns, (2) regional cancer awareness month campaigns, (3) targeted interventions, and (4) untargeted interventions. Most articles included in this review (16/23, 70\%) evaluated national cancer awareness month campaigns, defined as national campaigns to improve cancer awareness and screening delivered to the general public; Multimedia Appendix 3 details an example campaign. Two articles $(2 / 23,9 \%)$ evaluated regional cancer awareness month campaigns. These were campaigns that took place at the same time as national campaigns but were delivered to a specific region $[40,41]$. Three articles $(3 / 23,13 \%)$ evaluated targeted interventions that used defined activities to deliver cancer awareness and screening information to specific groups and were not part of a cancer awareness month campaign. Multimedia Appendix 4 provides an example of a targeted intervention. Two articles $(2 / 23,9 \%)$ evaluated untargeted interventions that included cancer awareness and screening information delivered to the general public that were not part of an awareness month campaign. One untargeted intervention article $(1 / 23,4 \%)$ explored the impact 
of a celebrity Tweet about having cancer and the test that saved their life [42]. The other untargeted intervention delivered cancer information videos to the public [43].

\section{Cancer Type}

Most commonly, the articles reported interventions aimed at breast cancer $(12 / 23,52 \%)$, followed by prostate and testicular cancer $(7 / 23,30 \%)$, cervical cancer $(4 / 23,17 \%)$ [44-47], colorectal cancer $(3 / 23,13 \%)$ [42,48,49], generic cancer (no specific cancer type; 3/23, 13\%) [50-52], familial cancer (inherited tumors; $1 / 23,4 \%)$, and lung cancer $(1 / 23,4 \%)$ $[42,43]$. Some articles evaluated interventions for more than one cancer type $(7 / 23,30 \%)$.

\section{Intervention Source}

Interventions were most commonly delivered by cancer charities $(17 / 23,74 \%)$, followed by public health government bodies $(4 / 23,17 \%)[40,44,47,53]$, regional health services $(2 / 23,9 \%)$ [43,54], a university $(1 / 23,4 \%)$ [43], and a celebrity $(1 / 23,4 \%)$ [42].

\section{Social Media Platform}

Interventions were most commonly delivered via Twitter (13/23, $57 \%)$, followed by Facebook $(8 / 23,35 \%)$, YouTube $(3 / 23,13 \%)$ [44,49,51], Instagram $(2 / 23,9 \%)$ [49,52], and Snapchat (1/23, 4\%) [54]. Some articles evaluated interventions delivered via more than one platform $(4 / 23,17 \%)$.

\section{Nature of Cancer Information Delivered and Shared in Interventions}

Just over one-half of the articles (13/23, 57\%) analyzed the messages that were delivered and shared by users and intervention developers. Several articles reported that most posts for national campaigns contained non-health messages and nonactionable messages [46,48,51,55-59]. During the 2013 Canadian Movember campaign, there were significantly more tweets on non-health topics, such as moustache growing $(n=3549)$, than on health topics $(n=673)$; only $0.6 \%(25 / 4222)$ of tweets analyzed were about cancer [56]. Furthermore, national campaigns heavily promoted online purchasing and fundraising to support cancer charities [55,57]. Bravo and Hoffman-Goetz [57] found that posts about fundraising and purchasing often did not mention cancer; only $2 \%$ (18/819) of fundraising tweets identified prostate or testicular cancer as the reason why they were fundraising. Additionally, for breast, cervical, prostate, and testicular cancer, gendered imagery and language were used to engage users $[45,55,57]$. In the Movember campaign, 9\% (204/2400) of tweets analyzed used war metaphors, with users describing themselves as an "army," and the moustaches characterized as being "manly" [57].

\section{Mechanisms of Behavior Change}

\section{BCTs Used in Interventions}

No articles reported theories that informed the development of the intervention. Table 2 details the 10 BCTs that we tentatively identified as being present in the interventions [38]. All interventions targeted cancer screening behaviors such as improving attendance at cancer screening. This was achieved by providing information on cancer screening but also by raising awareness via fundraising activities.

Table 2. The number of interventions that used possible behavior change techniques $(n=23)$ [38].

\begin{tabular}{ll}
\hline Behavior change techniques used in interventions & $\mathrm{n}(\%)$ \\
\hline Credible sources (eg, health professional, government, charity, celebrity) & $23(100)$ \\
Information about health consequences & $23(100)$ \\
Instruction on how to perform a behavior & $22(96)$ \\
Social support & $17(74)$ \\
Social comparison (eg, comparing a person's actions to the actions of others) & $17(74)$ \\
Information about others' approval & $17(74)$ \\
Goal-setting behavior & $16(70)$ \\
Social incentive (eg, providing a written reward only if a person performs the desired action) & $12(52)$ \\
Salience of consequences (eg, emphasizing the consequences of cancer) & $2(9)$ \\
Restructuring the physical environment (ie, changing the environment to facilitate the desired action) & $1(4)$
\end{tabular}

The main way in which interventions appeared to influence behavior change was by providing information about the health consequences of cancer, often by providing links to cancer charity/public health websites to access cancer symptom and screening information $[55,60]$. The information delivered by interventions was all from credible sources, such as government bodies or cancer charities, but information from users may have been less credible. Information was made salient to users in posts that mentioned how users and members of their family were at risk of cancer (eg, "your children and family depend on it”) [55,60]. Approximately three-quarters $(17 / 23,74 \%)$ of the articles reported interventions that used social support, social comparisons, and others' approval to encourage cancer screening. This was demonstrated by the sharing of personal stories and messages of support from the public, charities, organizations, cancer survivors, and celebrities [46,55,57]. One article reported an intervention that restructured the environment by improving access to booking breast screening appointments through Facebook Messenger [61]. Interventions also influenced behavior change through goal setting and providing incentives. 
For example, many campaigns encouraged users to complete fundraising goals, such as growing a moustache for Movember to raise awareness [55-58]. They used social incentives like congratulating and thanking users on social media for taking part in fundraising activities to promote cancer screening $[50,60]$.

\section{Methodological Approaches Used to Evaluate Interventions}

Multimedia Appendix 5 provides a breakdown of the methods used in each article. In most of the included articles (20/23, $87 \%$ ), quantitative research designs were used; one-quarter $(5 / 20,25 \%)$ of those were experimental research designs. A single-group posttest design was the most commonly used experimental design $(3 / 5,60 \%)[40,43,44]$. Two articles $(2 / 5$, $40 \%$ ) that used experimental designs used two-group pre- and posttest research designs [47,54]. Most (15/20, 75\%) of the quantitative articles used observational research designs, where the researchers observed the impact of previously developed interventions. Just over one-half $(8 / 15,53 \%)$ of these were longitudinal studies. One-half $(4 / 8,50 \%)$ of the longitudinal studies measured outcomes over a period of 1 to 2 years [50-53]. Two $(2 / 23,8.7 \%)$ articles used qualitative research designs $[55,57]$ and thematically analyzed social media messages. One $(1 / 23,4 \%)$ article used mixed methods by conducting a single-group pre- and posttest design and thematic analysis of social media messages [61].

\section{Outcomes Used}

Multimedia Appendix 5 shows the outcomes measured for each included article. Most $(18 / 23,78 \%)$ articles measured at least one of Neiger et al's [35] key performance indicators related to social media use in health promotion. Two $(2 / 23,9 \%)$ articles assessed insights by measuring user opinions $[40,43]$. Six $(6 / 23$, $26 \%$ ) articles measured exposure, the most common indicator being impressions (the number of times a post was viewed; 4/6, $67 \%)[40,41,49,62]$. Three $(3 / 23,13 \%)$ articles measured reach and the demographics of followers $[40,49,52]$. Five $(5 / 23,22 \%)$ articles measured low-level engagement by measuring the number of likes on a post $[40,46,49,50,53]$. Many $(11 / 23,48 \%)$ of the included articles measured medium-level engagement, with the most common indicator being the number of posts/tweets by users $(8 / 11,73 \%)$ [42,45,48,51,52,59,62]. Five articles $(5 / 23,22 \%)$ measured indicators of high-level engagement. Two $(2 / 5,40 \%)$ of these articles measured knowledge of cancer symptoms and screening [47,54] and one $(1 / 5,20 \%)$ article measured intention to get screened [40]. One $(1 / 5,20 \%)$ article measured participation in an offline advocacy event as a volunteer [41] and one $(1 / 5,20 \%)$ measured the number of people who attended screening [61].

\section{Key Article Findings}

\section{Insights, Exposure, and Reach}

Multimedia Appendix 5 presents the findings of each included article. Exposure varied by the type of intervention that was being evaluated. For example, one evaluation of a national campaign reported over 2 million Facebook impressions [49], and a regional campaign had 53,317 Facebook impressions [40]. Two $(2 / 23,9 \%)$ articles reported user insights and found social media was an acceptable way to deliver cancer awareness and screening information $[40,43]$. One $(1 / 23,4 \%)$ article found that $88 \%(43 / 49)$ of women surveyed indicated that they were neutral or agreed with seeing mammogram information on Facebook [40].

Three $(3 / 23,13 \%)$ articles suggested that reach varied by gender but only discussed the reach to women and men and no other gender identities. Content on YouTube may have reached more men than women, but Facebook content may have reached more women than men $[40,43,49]$. One $(1 / 23,4 \%)$ article found Facebook content had a wider reach to those aged 45 to 64 years than to other age groups [40]. Another $(1 / 23,4 \%)$ article suggested that campaigns tended to reach more White users (93\%) than African American (7\%) or Asian or Hispanic users $(0.6 \%)$ [49].

\section{Low-Level and Medium-Level Engagement}

One $(1 / 23,4 \%)$ article showed that users most commonly interacted with campaigns on Facebook by liking posts, followed by sharing content and commenting [50]. Two (2/23, 9\%) articles showed that social media influencers and celebrities increased the number of likes due to their large number of followers $[46,49]$. Three $(3 / 23,13 \%)$ articles found that retweeting was significantly more likely if the tweet was posted by celebrities, organizations, someone with a high number of followers, or someone who frequently tweeted about the campaign $[42,46,62]$. Three $(3 / 23,13 \%)$ articles reported that posts with images were the most liked and were more likely to be retweeted than posts with just text $[50,53,59]$.

Five $(5 / 23,22 \%)$ articles reported that engagement increased during the campaigns and decreased to baseline levels or below after the campaigns [41,50-52,62]. Two $(2 / 23,9 \%)$ articles found that health information-sharing tweets about cancer tended to rise during campaigns $[42,48]$. Two $(2 / 23,9 \%)$ articles reported that breast cancer campaigns had much more traffic on social media than other cancer campaigns, even on months that were dedicated to raising awareness of other cancers $[42,51]$. For example, even though the campaigns for prostate cancer awareness occurred in November, breast cancer received more mentions on Twitter in November than prostate cancer (284,015 posts versus 65,820 posts, respectively) [51]. Two $(2 / 23,9 \%)$ articles reported that colorectal cancer received the least attention on social media compared with breast, prostate, and cervical cancer [42,52]. Engagement with campaigns may vary by ethnicity, as one $(1 / 23,4 \%)$ article found White users consistently mentioned breast and prostate cancer more than other ethnicities [52].

\section{High-Level Engagement}

One $(1 / 23,4 \%)$ article reported that 9000 participants took part in an offline advocacy event that was part of a regional social media BCAM campaign [41]. Two $(2 / 23,9 \%)$ articles reported that targeted interventions improved knowledge of cancer symptoms and screening compared with a control [47,54]. One $(1 / 23,4 \%)$ article found that a regional mammography campaign improved intention to attend cancer screening; $82 \%$ of 49 women surveyed expressed an intent to get a mammogram in the next year [40]. One $(1 / 23,4 \%)$ targeted intervention reported 
an increase in the number of people who attended a breast screening appointment; attendance increased by an average of $12.9 \%$ across seven screening sites in North Midlands, United Kingdom (see Multimedia Appendix 4) [61].

\section{Discussion}

\section{Principal Findings}

Most studies of social media interventions have evaluated national cancer awareness month campaigns, using observational studies to measure exposure, reach, and low- to medium-level engagement with a campaign. A small number of studies suggested that regional cancer awareness month campaigns and targeted interventions might improve cancer awareness, as well as screening intentions and uptake. There was evidence that exposure, reach, and engagement with the interventions varied by age, gender, and ethnicity of users, and also by cancer type.

This scoping review was the first to focus on social media evaluations of interventions to improve cancer screening and early diagnosis. It added to the literature by highlighting the limited number of robust evaluations that captured high-level engagement/behavior change, such as attendance at cancer screening. Evaluating high-level engagement is challenging because timely observational data on cancer screening attendance can be difficult to access and link with social media data [24]. Evaluation is also challenging because social media interventions are often designed without evaluation in mind [3]. During the consultation for this review, experts commented that campaigns are set up so quickly that there is not always time to consider evaluation. Improving cancer screening and early diagnosis is seen as a long-term goal that will take many years to realize, so immediate changes might not be expected or measured. A comprehensive evaluation framework that incorporates elements from behavior change theories and social media engagement frameworks could foster more robust evaluations that capture outcomes that demonstrate impact on behavior change and engagement $[17,63,64]$. However, as noted during the consultation for this review, there are further challenges to evaluation, such as the difficulty of demonstrating that a specific campaign caused a change in outcomes, as well as the limited time and resources of organizations to conduct evaluations.

This review was the first to use the BCT taxonomy to identify a variety of BCTs that social media interventions used to change health behaviors, including social support and providing information about health consequences [38]. One article reported restructuring the environment to provide better access to cancer screening [61]. As noted during our consultation, governance and data protection issues often limit the ability of health providers to use social media to improve access to care. These issues need to be addressed if interventions are to be tested or implemented on a larger scale. Many articles also reported that information delivered and shared during national cancer awareness month campaigns consisted of more non-health messages relating to fundraising than health messages relating to cancer. For many of these campaigns, the theory of change may be that fundraising messages increase cancer awareness, thereby increasing help-seeking or uptake of screening. Behavior change theory suggests that providing actionable health messages, such as information on cancer symptoms, could influence behavior change more directly than fundraising messages $[12,38,63,65,66]$. Future evaluations are needed to test our assumptions about how national campaigns might lead to behavior change and what messages would be most effective.

We identified a need for more social media interventions targeted toward colorectal and lung cancer, as most of the articles in this review were evaluations of social media interventions for breast and prostate cancer awareness and screening. Survival rates for lung and colorectal cancer are poorer than for breast and prostate cancer, which is partly because of poor uptake of cancer screening and delayed help-seeking that can lead to a delayed diagnosis [67]. Previous studies $[68,69]$ have shown that there is more stigma around lung cancer than other cancers, and higher perceptions of cancer stigma are associated with delays in seeking medical care. Social media interventions could play a key role in changing social norms and stigma around help-seeking and screening for these cancers [70]. BCTs used by current breast cancer campaigns, such as sharing personal stories, could help to create social support and influence how people view these cancers, which in turn could encourage help-seeking behavior, increase screening uptake, and improve health outcomes.

This review also added to the literature by exploring to what extent inequalities in cancer screening and early diagnosis were measured and potentially addressed by social media interventions. We found some evidence that social media interventions have poorer reach and engagement with ethnic minorities, but there was no information on engagement with other minority groups [52]. Individuals from ethnic minorities might have less interaction with social media campaigns and not seek out cancer screening information because they have less access to cancer screening and higher cancer stigma [68,71-74]. The written and visual communication in social media interventions may also exclude ethnic minorities if the information is only available in English, presented in inaccessible language, or framed in a way that is unrelatable [74]. A lack of social media influencers or campaign role models that resonate with ethnic minorities may also make it less likely that they undertake a behavior, as suggested by social cognitive theory [75]. Future evaluations of social media interventions should measure inequalities in exposure, reach, and engagement and consider their success directly in relation to the groups that they were seeking to target. Targeting interventions toward those with a disproportionate disease burden could help to improve health inequalities seen in cancer screening and early diagnosis. However, social media interventions will have little impact on those who do not use social media, who may also be those in greatest need of information on cancer screening.

Some differences in the use of social media platforms by gender and age were found in this review [49,62]. Facebook content reached more women than men, and reached older-aged adults, and YouTube content reached more men than women [40,43,49]. This is consistent with recent data on social media use in the United Kingdom by age and gender [76]. This highlights the importance of identifying which platforms target users are currently more likely to engage with when designing social 
media interventions. However, as noted during our consultation, social media is a constantly changing landscape, so messaging needs to be continually updated and transferred to different platforms. Targeting messages also has potential risks, such as the threat to privacy and ethical issues, and it often requires payment and significant time and resources [17,24]. Additionally, data may not be available for particular target users; for example, we only found evidence to show how best to target those who identify as male and female. Previous research has shown that there are gender identity disparities in cancer screening, and trans and nonbinary individuals could benefit from more information regarding cancer screening and early diagnosis [77,78]. Further research is needed to understand the most effective way of targeting social media interventions toward these individuals.

\section{Limitations}

We acknowledge that scoping reviews have several limitations, but a scoping review allowed us to gain a wide-ranging understanding of the role of social media in cancer screening. Research into social media is rapidly growing and this scoping review is a snapshot of evidence for social media interventions at a particular time [26]. Furthermore, as we only selected studies written in English that did not include information about some popular social networking sites in non-English-speaking countries, the findings of this review might not be generalizable. Many of the social media campaigns in this review were also part of multimedia campaigns. Therefore, it is difficult to know whether changes in engagement with social media or health behaviors were due to the social media element of those campaigns and how social media interacts with other aspects of the campaign. Additionally, the coding of BCTs in this review was dependent on reported content and online sources, so there was insufficient detail to identify all techniques used. The review was also dependent on what outcomes the evaluations chose to measure and report. There is currently no protocol for how to report evaluations of social media interventions, so there may be some degree of reporting bias in included articles. Future research would benefit from the development of a reporting protocol based on current frameworks for evaluating social media research [35].

\section{Conclusions}

This review found that most evaluations of social media interventions to improve cancer screening and early diagnosis did not report behavior change outcomes. The limited available evidence suggests some types of social media interventions may improve cancer awareness and intended and actual uptake of screening. Use of evaluation frameworks and reporting guidelines could help future researchers to plan robust evaluations of social media interventions that capture outcomes of behavior change and explore how these interventions work. Future evaluations could also measure who engaged with these interventions to assess whether social media interventions for cancer screening and early diagnosis can address some health inequalities. Interventions focusing on cancers that have received less social media attention, such as colorectal and lung cancer, could help to influence social norms around help-seeking and screening uptake for these cancers, which could improve health outcomes for patients.

\section{Acknowledgments}

We would like to thank Veronica Parisi for contributing to the search strategy, and Sali Rizahu for contributing to the title/abstract screening. We are indebted to Dr Julie Peconi, Kate Sanger, Caroline Bravo, Peter Nuckley, Gina Newman, and Jessica Mulroy-Johnson for consulting with us on our preliminary findings and commenting on the paper. This report presents independent research commissioned and funded by the National Institute for Health Research (NIHR) Policy Research Program, conducted through the Policy Research Unit in Cancer Awareness, Screening and early Diagnosis, PR-PRU-1217-21601. RP is supported by the NIHR School for Public Health Research (Grant Reference Number PD-SPH-2015). JS is supported by the National Institute for Health Research Applied Research Collaboration (ARC) North Thames. The views expressed in this publication are those of the authors and not necessarily those of the NIHR or the Department of Health and Social Care or its arm's length bodies, or other government departments. The funders had no involvement in review or approval of the manuscript.

\section{Authors' Contributions}

AK, APK, and AC were involved in the screening of titles and abstracts, as well as the full-text screening. RP, APK, and AC were responsible for data extraction. CVW, JW, JS, APK, and AK were involved in the design of the study. RP analyzed the data, and all authors contributed to the interpretation of the results and reviewed and approved the manuscript for publication.

\section{Conflicts of Interest}

None declared.

\section{Multimedia Appendix 1}

MEDLINE search strategy.

[DOCX File, 13 KB-Multimedia Appendix 1]

\section{Multimedia Appendix 2}

Characteristics of included articles and interventions ordered by social media platform and target cancer of interventions. 
[DOCX File , 18 KB-Multimedia Appendix 2]

\section{Multimedia Appendix 3}

Description of the Movember campaign.

[DOCX File, 17 KB-Multimedia Appendix 3]

\section{Multimedia Appendix 4}

Description of a targeted media intervention using Facebook.

[DOCX File, 14 KB-Multimedia Appendix 4]

\section{Multimedia Appendix 5}

Characteristics of the evaluations ordered by research design, including sampling, duration, outcome, and key findings. [DOCX File, 26 KB-Multimedia Appendix 5]

\section{References}

1. Internet access - households and individuals, Great Britain. Office for National Statistics website. 2018. URL: https://www. ons.gov.uk/peoplepopulationandcommunity/householdcharacteristics/homeinternetandsocialmediausage/bulletins/ internetaccesshouseholdsandindividuals/2018

2. Kemp S. Digital 2020: Global Digital Overview. DataReportal. 2020. URL: https://datareportal.com/reports/ digital-2020-global-digital-overview

3. Korda H, Itani Z. Harnessing social media for health promotion and behavior change. Health Promot Pract 2013 Jan;14(1):15-23. [doi: 10.1177/1524839911405850] [Medline: 21558472]

4. Neiger BL, Thackeray R, Burton SH, Thackeray CR, Reese JH. Use of twitter among local health departments: an analysis of information sharing, engagement, and action. J Med Internet Res 2013 Aug; 15(8):e177 [FREE Full text] [doi: 10.2196/jmir.2775] [Medline: 23958635]

5. Wakefield MA, Loken B, Hornik RC. Use of mass media campaigns to change health behaviour. Lancet 2010 Oct 9;376(9748):1261-1271 [FREE Full text] [doi: 10.1016/S0140-6736(10)60809-4] [Medline: 20933263]

6. Jackson D. Sprout Social. How to Go Viral Steps to Reach a Massive Audience. 2015. URL: https://sproutsocial.com/ insights/how-to-go-viral/ [accessed 2020-06-19]

7. Smith BG, Kendall MC, Knighton D, Wright T. Rise of the Brand Ambassador: Social Stake, Corporate Social Responsibility and Influence among the Social Media Influencers. CMR 2018 Jul 30;03(01):6-29. [doi: 10.22522/cmr20180127]

8. Welch V, Petkovic J, Pardo PJ, Rader T, Tugwell P. Interactive social media interventions to promote health equity: an overview of reviews. Health Promot Chronic Dis Prev Can 2016 Apr;36(4):63-75 [FREE Full text] [Medline: 27077792]

9. Cavallo DN, Chou WS, McQueen A, Ramirez A, Riley WT. Cancer prevention and control interventions using social media: user-generated approaches. Cancer Epidemiol Biomarkers Prev 2014 Sep;23(9):1953-1956. [doi:

10.1158/1055-9965.EPI-14-0593] [Medline: 25103820]

10. Aiello AE, Renson A, Zivich PN. Social Media- and Internet-Based Disease Surveillance for Public Health. Annu. Rev. Public Health 2020 Apr 02;41(1):101-118. [doi: 10.1146/annurev-publhealth-040119-094402]

11. Gandomi A, Haider M. Beyond the hype: Big data concepts, methods, and analytics. International Journal of Information Management 2015 Apr;35(2):137-144. [doi: 10.1016/j.ijinfomgt.2014.10.007]

12. Gough A, Hunter RF, Ajao O, Jurek A, McKeown G, Hong J, et al. Tweet for Behavior Change: Using Social Media for the Dissemination of Public Health Messages. JMIR Public Health Surveill 2017 Mar 23;3(1):e14 [FREE Full text] [doi: 10.2196/publichealth.6313] [Medline: 28336503]

13. About Be Clear on Cancer. Cancer Research UK. 2014. URL: https://www.cancerresearchuk.org/health-professional/ awareness-and-prevention/be-clear-on-cancer/about-be-clear-on-cancer

14. Breast Cancer Awareness Month. National Breast Cancer Foundation. 2020. URL: https://www.nationalbreastcancer.org/ breast-cancer-awareness-month

15. Peacock O, Clayton S, Atkinson F, Tierney GM, Lund JN. 'Be Clear on Cancer': the impact of the UK National Bowel Cancer Awareness Campaign. Colorectal Dis 2013 Aug;15(8):963-967. [doi: 10.1111/codi.12220] [Medline: 23656572]

16. Bethune R, Marshall MJ, Mitchell SJ, Oppong C, Cartmel MT, Arumugam PJ, et al. Did the 'Be Clear on Bowel Cancer' public awareness campaign pilot result in a higher rate of cancer detection? Postgrad Med J 2013 Jul;89(1053):390-393. [doi: 10.1136/postgradmedj-2012-131014] [Medline: 23572594]

17. Dunn AG, Mandl KD, Coiera E. Social media interventions for precision public health: promises and risks. NPJ Digit Med 2018;1:47 [FREE Full text] [doi: 10.1038/s41746-018-0054-0] [Medline: 30854472]

18. Whyte-Smith H, Carpenter R, Koray E, Roe H. Technology Tracker Q2 2018. Ipsos. 2018. URL: https://www.ipsos.com/ sites/default/files/ct/publication/documents/2019-03/tech tracker q2 2018 final.pdf 
19. Knoll J. Advertising in social media: a review of empirical evidence. International Journal of Advertising $2015 \mathrm{Mar}$ 24;35(2):266-300. [doi: 10.1080/02650487.2015.1021898] [Medline: 000371744200007]

20. Chou WYS, Hunt YM, Beckjord EB, Moser RP, Hesse BW. Social media use in the United States: implications for health communication. J Med Internet Res 2009 Nov 27;11(4):e48 [FREE Full text] [doi: 10.2196/jmir.1249] [Medline: 19945947]

21. Shah R. Fake news: A threat to curbing vaccine-preventable diseases in the UK. Nuffield Trust. 2019. URL: https://www. nuffieldtrust.org.uk/news-item/fake-news-a-threat-to-curbing-vaccine-preventable-diseases-in-the-uk

22. Moving the Needle: Promoting vaccination uptake across the life course. Royal Society for Public Health. 2019. URL: https://www.rsph.org.uk/static/uploaded/3b82db00-a7ef-494c-85451e78ce18a779.pdf

23. Health and Digital: Reducing Inequalities, Improving Society. Tinder Foundation. 2016. URL: https://www.basw.co.uk/ system/files/resources/basw_54514-4_0.pdf

24. Pagoto S, Waring ME, Xu R. A Call for a Public Health Agenda for Social Media Research. J Med Internet Res 2019 Dec 19;21(12):e16661. [doi: 10.2196/16661] [Medline: $\underline{\text { 31855185] }}$

25. Schliemann D, Su TT, Paramasivam D, Treanor C, Dahlui M, Loh SY, et al. Effectiveness of Mass and Small Media Campaigns to Improve Cancer Awareness and Screening Rates in Asia: A Systematic Review. J Glob Oncol 2019 Apr;5:1-20 [FREE Full text] [doi: 10.1200/JGO.19.00011] [Medline: $\underline{\text { 30969807] }}$

26. Koskan A, Klasko L, Davis SN, Gwede CK, Wells KJ, Kumar A, et al. Use and taxonomy of social media in cancer-related research: a systematic review. Am J Public Health 2014 Jul;104(7):e20-e37 [FREE Full text] [doi:

10.2105/AJPH.2014.301980] [Medline: 24832403]

27. Tricco AC, Lillie E, Zarin W, O'Brien K, Colquhoun H, Kastner M, et al. A scoping review on the conduct and reporting of scoping reviews. BMC Med Res Methodol 2016 Feb 09;16:15 [FREE Full text] [doi: 10.1186/s12874-016-0116-4] [Medline: 26857112]

28. Arksey H, O'Malley L. Scoping studies: towards a methodological framework. International Journal of Social Research Methodology 2005 Feb;8(1):19-32. [doi: 10.1080/1364557032000119616]

29. Levac D, Colquhoun H, O'Brien KK. Scoping studies: advancing the methodology. Implement Sci 2010;5:69 [FREE Full text] [doi: 10.1186/1748-5908-5-69] [Medline: 20854677]

30. Peters MDJ, Godfrey CM, Khalil H, McInerney P, Parker D, Soares CB. Guidance for conducting systematic scoping reviews. Int J Evid Based Healthc 2015 Sep;13(3):141-146. [doi: 10.1097/XEB.0000000000000050] [Medline: 26134548]

31. Tricco AC, Lillie E, Zarin W, O'Brien KK, Colquhoun H, Levac D, et al. PRISMA Extension for Scoping Reviews (PRISMA-ScR): Checklist and Explanation. Ann Intern Med 2018 Oct 02;169(7):467-473. [doi: 10.7326/M18-0850] [Medline: $\underline{30178033}$ ]

32. Kaushal A, Kassianos AP, Sheringham J, Waller J, von Wagner C. Use of social media for cancer prevention and early diagnosis: scoping review protocol. BMJ Open 2020 Feb 25;10(2):e033592. [doi: 10.1136/bmjopen-2019-033592]

33. The History of Social Media. Future Marketing. 2017. URL: https://www.future-marketing.co.uk/the-history-of-social-media/

34. Cohen J. A Coefficient of Agreement for Nominal Scales. Educational and Psychological Measurement 1960;20(1):37-46. [doi: 10.1177/001316446002000104] [Medline: 5637250]

35. Neiger BL, Thackeray R, Van WSA, Hanson CL, West JH, Barnes MD, et al. Use of social media in health promotion: purposes, key performance indicators, and evaluation metrics. Health Promot Pract 2012 Mar;13(2):159-164. [doi: 10.1177/1524839911433467] [Medline: 22382491]

36. Fishbein M, Yzer MC. Using Theory to Design Effective Health Behavior Interventions. Commun Theory 2003 May;13(2):164-183. [doi: 10.1111/j.1468-2885.2003.tb00287.x]

37. Simon AE, Waller J, Robb K, Wardle J. Patient Delay in Presentation of Possible Cancer Symptoms: The Contribution of Knowledge and Attitudes in a Population Sample from the United Kingdom. 2010 Jul 26;19(9):2272-2277. [doi: 10.1158/1055-9965.epi-10-0219]

38. Michie S, Richardson M, Johnston M, Abraham C, Francis J, Hardeman W, et al. The behavior change technique taxonomy (v1) of 93 hierarchically clustered techniques: building an international consensus for the reporting of behavior change interventions. Ann Behav Med 2013 Aug;46(1):81-95. [doi: 10.1007/s12160-013-9486-6] [Medline: 23512568]

39. Popay J, Roberts H, Sowden A, Petticrew M, Arai L, Rodgers M. Guidance on the Conduct of Narrative Synthesis in Systematic Reviews : a product of the ESRC methods programme (Version 1).: Lancaster, UK: University of Lancaster; 2006. URL: https://www.lancaster.ac.uk/media/lancaster-university/content-assets/documents/fhm/dhr/chir/ NSsynthesisguidanceVersion1-April2006.pdf [accessed 2020-10-30]

40. Klippert H, Schaper A. Using Facebook to communicate mammography messages to rural audiences. Public Health Nurs 2018 Oct 29;36(2):164-171. [doi: 10.1111/phn.12556]

41. Salako O, Roberts AA, Isibor VI, Babatunde O, Fatiregun O, Nwogu CN. Innovative Breast Cancer Awareness and Advocacy Campaign. JGO 2017 Apr;3(2):169-176. [doi: 10.1200/jgo.2016.003509]

42. Vos SC, Sutton J, Gibson CB, Butts CT. Celebrity Cancer on Twitter: Mapping a Novel Opportunity for Cancer Prevention. Cancer Control 2019 Feb 28;26(1):107327481982582. [doi: 10.1177/1073274819825826]

43. Jones GE, Singletary JH, Cashmore A, Jain V, Abhulimhen J, Chauhan J, et al. Developing and assessing the utility of a You-Tube based clinical genetics video channel for families affected by inherited tumours. Familial Cancer 2016 Jan 11;15(2):351-355. [doi: $\underline{10.1007 / \mathrm{s} 10689-016-9866-8]}$ 
44. Cooper CP, Gelb CA, Chu J. Gynecologic Cancer Information on YouTube: Will Women Watch Advertisements to Learn More? J Cancer Educ 2016 Sep;31(3):602-604 [FREE Full text] [doi: 10.1007/s13187-015-0830-3] [Medline: 25877466]

45. Lenoir P, Moulahi B, Azé J, Bringay S, Mercier G, Carbonnel F. Raising Awareness About Cervical Cancer Using Twitter: Content Analysis of the 2015 \#SmearForSmear Campaign. J Med Internet Res 2017 Oct 16;19(10):e344. [doi: 10.2196/jmir.8421]

46. Teoh D, Shaikh R, Vogel RI, Zoellner T, Carson L, Kulasingam S, et al. A Cross-Sectional Review of Cervical Cancer Messages on Twitter During Cervical Cancer Awareness Month. Journal of Lower Genital Tract Disease 2018;22(1):8-12. [doi: $10.1097 /$ lgt.0000000000000363]

47. Lai C, Wu W, Tsai S, Cheng S, Lin K, Liang S. The Effectiveness of a Facebook-Assisted Teaching Method on Knowledge and Attitudes About Cervical Cancer Prevention and HPV Vaccination Intention Among Female Adolescent Students in Taiwan. Health Educ Behav 2014 Dec 15;42(3):352-360. [doi: 10.1177/1090198114558591]

48. Lee KC, Oh H, Park G, Park S, Suh B, Bae WK, et al. Transmissibility of the Campaign for Colorectal Cancer Awareness in Korea Among Twitter Users. Ann Coloproctol 2016 Oct;32(5):184-189 [FREE Full text] [doi: 10.3393/ac.2016.32.5.184] [Medline: 613299883]

49. \#MyBest10Seconds ECCAM2019 DiCE Campaign Report. Digestive Cancers Europe. Europa Colon;. 2019 Apr. URL: https://issuu.com/diceeurope/docs/_mybest10seconds_campaign_report_5dfcbfe1b2922b

50. Fernández-Gómez E, Díaz-Campo J. Comunicación sobre el cáncer en Facebook. Las asociaciones de Argentina, Chile, Colombia y España. cuadernos.info 2016 May 05(38):35-50. [doi: 10.7764/cdi.38.926]

51. Vraga EK, Stefanidis A, Lamprianidis G, Croitoru A, Crooks AT, Delamater PL, et al. Cancer and Social Media: A Comparison of Traffic about Breast Cancer, Prostate Cancer, and Other Reproductive Cancers on Twitter and Instagram. Journal of Health Communication 2018 Jan 09;23(2):181-189. [doi: 10.1080/10810730.2017.1421730]

52. Xu S, Markson C, Costello KL, Xing CY, Demissie K, Llanos AA. Leveraging Social Media to Promote Public Health Knowledge: Example of Cancer Awareness via Twitter. JMIR Public Health Surveill 2016;2(1):e17 [FREE Full text] [doi: 10.2196/publichealth.5205] [Medline: 27227152]

53. Theiss SK, Burke RM, Cory JL, Fairley TL. Getting beyond impressions: an evaluation of engagement with breast cancer-related Facebook content. mHealth 2016 Nov 07;2:41-41 [FREE Full text] [doi: 10.21037/mhealth.2016.10.02] [Medline: 27840816]

54. Alanzi TM, Alobrah A, Alhumaidi R, Aloraifi S. Evaluation of the SnapChat mobile social networking application for breast cancer awareness among Saudi students in the Dammam Region of the Kingdom of Saudi Arabia. BCTT 2018 Jul; Volume 10:113-119. [doi: 10.2147/bctt.s166135]

55. Abramson K, Keefe B, Chou WS. Communicating about cancer through Facebook: a qualitative analysis of a breast cancer awareness page. J Health Commun 2015 Dec;20(2):237-243. [doi: 10.1080/10810730.2014.927034] [Medline: 25495200]

56. Bravo CA, Hoffman-Goetz L. Tweeting About Prostate and Testicular Cancers: Do Twitter Conversations and the 2013 Movember Canada Campaign Objectives Align? J Cancer Educ 2016 Jun;31(2):236-243. [doi: 10.1007/s13187-015-0796-1] [Medline: 25649663]

57. Bravo CA, Hoffman-Goetz L. Tweeting About Prostate and Testicular Cancers: What Are Individuals Saying in Their Discussions About the 2013 Movember Canada Campaign? J Cancer Educ 2016 Sep;31(3):559-566. [doi: 10.1007/s13187-015-0838-8] [Medline: 25903054]

58. Bravo CA, Hoffman-Goetz L. Social Media and Men's Health: A Content Analysis of Twitter Conversations During the 2013 Movember Campaigns in the United States, Canada, and the United Kingdom. Am J Mens Health 2017

Nov;11(6):1627-1641. [doi: 10.1177/1557988315617826] [Medline: 26669771]

59. Chung JE. Retweeting in health promotion: Analysis of tweets about Breast Cancer Awareness Month. Computers in Human Behavior 2017 Sep;74:112-119. [doi: 10.1016/j.chb.2017.04.025]

60. Diddi P, Lundy LK. Organizational Twitter Use: Content Analysis of Tweets during Breast Cancer Awareness Month. Journal of Health Communication 2017 Feb 19;22(3):243-253. [doi: 10.1080/10810730.2016.1266716]

61. Using Facebook to improve attendance at screening appointments. Good Things Foundation. 2018. URL: https://drive. google.com/file/d/1pASaOsKqsJ5AEdwT3-xAzUbZFIDI4W7K/view

62. Thackeray R, Burton SH, Giraud-Carrier C, Rollins S, Draper CR. Using Twitter for breast cancer prevention: an analysis of breast cancer awareness month. BMC Cancer 2013 Oct 29;13:508 [FREE Full text] [doi: 10.1186/1471-2407-13-508] [Medline: 24168075]

63. Adewuyi E, Adefemi K. Behavior Change Communication Using Social Media: A Review. International Journal of Communication and Health 2016;9:109-116 [FREE Full text]

64. Althoff T, Jindal P, Leskovec J. Online Actions with Offline Impact: How Online Social Networks Influence Online and Offline User Behavior. 2017 Feb Presented at: Tenth ACM International Conference on Web Search and Data Mining; February, 2017; Cambridge United Kingdom p. 537-546 URL: http://europepmc.org/abstract/MED/28345078 [doi: $10.1145 / 3018661.3018672]$

65. Briscoe C, Aboud F. Behaviour change communication targeting four health behaviours in developing countries: a review of change techniques. Soc Sci Med 2012 Aug;75(4):612-621. [doi: 10.1016/j.socscimed.2012.03.016] [Medline: 22541798] 
66. Freeman B, Potente S, Rock V, McIver J. Social media campaigns that make a difference: what can public health learn from the corporate sector and other social change marketers? Public Health Res Pract 2015 Mar 30;25(2):e2521517 [FREE Full text] [doi: 10.17061/phrp2521517] [Medline: 25848735]

67. Redaniel M, Martin R, Ridd M, Wade J, Jeffreys M. Diagnostic intervals and its association with breast, prostate, lung and colorectal cancer survival in England: historical cohort study using the Clinical Practice Research Datalink. PLoS One 2015;10(5):e0126608 [FREE Full text] [doi: 10.1371/journal.pone.0126608] [Medline: 25933397]

68. Marlow LAV, Waller J, Wardle J. Does lung cancer attract greater stigma than other cancer types? Lung Cancer 2015 Apr;88(1):104-107. [doi: 10.1016/j.lungcan.2015.01.024] [Medline: 25704958]

69. Carter-Harris L, Hermann CP, Schreiber J, Weaver MT, Rawl SM. Lung cancer stigma predicts timing of medical help-seeking behavior. Oncol Nurs Forum 2014 May;41(3):E203-E210 [FREE Full text] [doi: 10.1188/14.ONF.E203-E210] [Medline: 24769603]

70. Metwally O, Blumberg S, Ladabaum U, Sinha SR. Using Social Media to Characterize Public Sentiment Toward Medical Interventions Commonly Used for Cancer Screening: An Observational Study. J Med Internet Res 2017 Jun 07;19(6):e200 [FREE Full text] [doi: 10.2196/jmir.7485] [Medline: 28592395]

71. Vrinten C, Gallagher A, Waller J, Marlow LAV. Cancer stigma and cancer screening attendance: a population based survey in England. BMC Cancer 2019 Jun 11;19(1):566 [FREE Full text] [doi: 10.1186/s 12885-019-5787-x] [Medline: 31185949]

72. Hirst Y, Stoffel S, Baio G, McGregor L, von Wagner C. Uptake of the English Bowel (Colorectal) Cancer Screening Programme: an update 5 years after the full roll-out. Eur J Cancer 2018 Nov; 103:267-273 [FREE Full text] [doi: 10.1016/j.ejca.2018.07.135] [Medline: 30196989]

73. Singh G, Jemal A. Socioeconomic and Racial/Ethnic Disparities in Cancer Mortality, Incidence, and Survival in the United States, 1950-2014: Over Six Decades of Changing Patterns and Widening Inequalities. J Environ Public Health 2017;2017:2819372 [FREE Full text] [doi: 10.1155/2017/2819372] [Medline: 28408935]

74. Viswanath K, Emmons K. Message Effects and Social Determinants of Health: Its Application to Cancer Disparities. Journal of Communication 2006 Aug 04;56(suppl_1):S238-SS64 [FREE Full text] [doi: 10.1111/j.1460-2466.2006.00292.x]

75. Bandura A. Social Cognitive Theory of Mass Communication. Media Psychology 2001 Aug;3(3):265-299. [doi: 10.1207/S1532785XMEP0303 03]

76. Adults: Media Use and Attitudes Report 2019. Ofcom. 2019 May 30. URL: https://www.ofcom.org.uk/ data/assets/ pdf_file/0021/149124/adults-media-use-and-attitudes-report.pdf

77. Tabaac AR, Sutter ME, Wall CSJ, Baker KE. Gender Identity Disparities in Cancer Screening Behaviors. Am J Prev Med 2018 Mar;54(3):385-393. [doi: 10.1016/j.amepre.2017.11.009] [Medline: 29338956]

78. Connolly D, Hughes X, Berner A. Barriers and facilitators to cervical cancer screening among transgender men and non-binary people with a cervix: A systematic narrative review. Prev Med 2020 Jun;135:106071. [doi: 10.1016/j.ypmed.2020.106071] [Medline: 32243938]

\section{Abbreviations}

BCAM: breast cancer awareness month

BCT: behavior change technique

CINAHL: Cumulative Index to Nursing and Allied Health Literature

PRISMA-ScR: Preferred Reporting Items for Systematic reviews and Meta-Analyses extension for Scoping Reviews

Edited by C Hoving; submitted 19.06.20; peer-reviewed by C Basch, T Gültzow; comments to author 27.07.20; revised version received
11.09.20; accepted 01.10.20; published 09.11.20
Please cite as:
Plackett R, Kaushal A, Kassianos AP, Cross A, Lewins D, Sheringham J, Waller J, von Wagner C
Use of Social Media to Promote Cancer Screening and Early Diagnosis: Scoping Review
J Med Internet Res 2020;22(11):e21582
URL: $\underline{\text { http://www.jmir.org/2020/11/e21582/ }}$
doi: $\underline{10.2196 / 21582}$
PMID: $\underline{3164907}$

CRuth Plackett, Aradhna Kaushal, Angelos P Kassianos, Aaron Cross, Douglas Lewins, Jessica Sheringham, Jo Waller, Christian von Wagner. Originally published in the Journal of Medical Internet Research (http://www.jmir.org), 09.11.2020. This is an open-access article distributed under the terms of the Creative Commons Attribution License (https://creativecommons.org/licenses/by/4.0/), which permits unrestricted use, distribution, and reproduction in any medium, 
provided the original work, first published in the Journal of Medical Internet Research, is properly cited. The complete bibliographic information, a link to the original publication on http://www.jmir.org/, as well as this copyright and license information must be included. 